In der Folge (1) gibt es ein $f_{s}$ so, daß

$$
\left|f_{s}(p)-\mu_{1} F^{\prime} \mu(p)\right|<\frac{\varepsilon}{2} . \quad p \in Q_{0}
$$

ist. Wir behaupten, da $B$

$$
\left|\Phi_{s}(p)-F(p)\right|<\varepsilon \quad p \in K^{(n)}
$$

ist. Es ist namlich $\mu_{1}(p) \in Q_{0}$ also $\psi^{s} \mu_{1}(p) \epsilon J_{s}$.

Laut (2) und (1 $\left.1^{a}\right)$ ist daher

$$
\Phi_{s}(p)=\mu f_{s} \mu_{1}(p) \text {. }
$$

Dies gibt aber, mit Rucksicht anf (3) und (4) die gewunschte Ungleichung (5).

Dieser Satz, angewendet fur $n=1$, ergibt die Lösung des am Anfang dieser Arbeit gestellten Problems:

Es gibt funf Funktionen $\left(\mu(x), \mu_{1}(x), \psi(x), \psi^{-1}(x), \chi(x)\right)$ so daß jede andere im Intervall $\langle 0,1\rangle$ erklärte, stetige Funktion, deren Werte demselben Intervall angehören, durch Zusammensetzen dieser fünf Funktionen, beliebig genau approximiert werden kann.

\section{Sur l'approximation des fonctions continues par} les superpositions de quatre fonctions.

Par

$$
\text { W. Si erpiński (Varsovie). }
$$

MM. J. Schreier et S. Ulam ont démontré dans ce volume un théorème, dont un cas particulier (pour l'espace linéaire) est la proposition suivante:

Il existe cinq fonctions definies et continues dans lintervalle I $(0 \leqslant x \leqslant 1)$ et dont les valeurs appartiennent à $I$, telles que toute autre fonction de même nature peut être approximée aussi près que l'on veut par. une superposition (finie) de ces cinq fonctions.

Le but de cette Note est de donner une démonstration directe de cette proposition (même en y remplaçant lo nombre 5 par 4). Nous la déduirons immédiatement $\mathrm{du}_{4}$ lemme suivant:

Lemme: $f_{1}(x), f_{3}(x), f_{3}(x), \ldots$ étant une suite infinie donnée de fonctions continues, définies dans lintervalle $I(0 \leqslant x \leqslant 1)$ et ne prenant que des valeurs de cet intervalle, il existe quatre fonctions: de même nature, telles que toute fonction de la suite infinie considerée est une superpasition (finie) de ces quatre fonctions.

Démonstration. Définissons dans $I$ les fonctions $\varphi(x), \vartheta(x)$ et $\chi(x)$ par les formules

(1) $\left\{\begin{array}{c}\varphi(x)=4 x \text { pour } 0 \leqslant x<1, \quad \varphi(x)=1 \text { pour } 1 \leqslant x \leqslant 1, \\ \vartheta(x)=\frac{x}{4}, \quad \chi(x)=\frac{x+1}{2}\end{array}\right.$

et définissons dans $I$ la fonction $\psi(x)$ comme il suit. 
Posons $\psi(0)=0$, et, si $n$ est un nombre naturel, posons

$$
\psi(x)=\frac{1}{2^{2 n-2}} f_{n}\left(2^{2 n-1} x-1\right) \text { pour } \frac{1}{2^{2 n-1}} \leqslant x \leqslant \frac{1}{2^{2 n-2}}
$$

$\psi(x)=\left[4 f_{n}(0)-f_{n+1}(1)\right] x+\frac{f_{n+1}(1)}{2^{2 n-1}}-\frac{f_{n}(0)}{2^{2 n-2}}$ pour $\frac{1}{2^{n}}<x<\frac{1}{2^{2 n-1}}$

Les fonctions $f_{n}(x)(n=1,2, \ldots)$ étant continues dans $I$ et ne prenant que des valeurs de cet intervalle, on vérifie sans peine que la fonction $\psi(x)$ est continue dans l'intervalle $I$.

Soit maintenant $n$ un nombre naturel donné. Je dis que (pour $0 \leqslant x \leqslant 1)$

(4)

$$
\left.f_{n}(x)=\varphi^{n-1} \psi \vartheta^{n-1} \chi(x)^{1}\right) .
$$

En effet, soit $x$ un nombre de l'intervalle 1 . On a donc, d'après (1):

$$
\vartheta^{n-1}(\chi(x))=\frac{x+1}{2^{2 n-1}}
$$

donc, d'après $0 \leqslant x \leqslant 1$

$$
\frac{1}{2^{2 n-1}} \leqslant \vartheta^{n-1} \chi(x) \leqslant \frac{1}{2^{2 n-2}}
$$

ce qui donne, d'après (2) et (5):

$$
\psi \vartheta^{n-1} \chi(x)=\frac{1}{2^{2 n-2}} f_{n}\left(2^{2 n-1} \vartheta^{n-1} \chi(x)-1\right)=\frac{1}{2^{2 n-2}} f_{n}(\dot{x}),
$$

d'où, d'après (1), résulto la formule (4).

Notre lemme est ainsi démontré. Pour en déduire la proposition de MM. Schreier et Ulam, il suffit de prendre pour $f_{n}(x)$ $(n=1,2, \ldots)$ une suite infinie formée de tous les polynomes en $x$ (définis dans $I$ ) réduits à l'intervalle $(0,1)^{2}$ ) aux coefficients rationnels et de faire appel au théorème bien connu de Weierstrass sur l'approximation de fonctions continues par les polynomes.

1) $g^{k}(x)$ dóigne la $k$-ième itération de la fonction $g(x)$ et $g^{0}(x)=x$.

3) c. a. d. tout polynome $P(x)$ doit être remplàcé par la fonction $F(x)$ ogale a $P(x)$, si $0 \leqslant P(x) \leqslant 1$, égale à 0 , si $P(x)<0$ et égale à 1 , ai $P(x)>1$.

\section{Sur les ensembles jouissant de la propriété} de Baire.

(Solution d'un problème de M. Szpilrajn).

Par

\section{W. Sierpiński (Varsovie).}

M. E. Szpilrajn a posé récemment le problème suivant.

Divisons tous les ensembles linéaires jouissant de la propriété de Baire ${ }^{1}$ ) en classes, en rangeant en une même classe deux ensembles dans ce et seulement dans ce cas, s'il ne différent que d'un ensemble toujours de première catégorie ${ }^{2}$ ).

Quelle est la puissance de la famille $F$ de ces classes?

En admettant que $2^{\mathrm{N}_{0}}=\aleph_{1}$ je prouverai que la famille $F$ est de puissance $2^{2^{\mathrm{N}_{0}}}$.

Démonstration. Admettons que $2^{x_{0}}=\aleph_{1}$. Dans ce cas il existe une suite transfinie du type $Q$,

$$
P_{1}, P_{2}, P_{3}, \ldots, P_{\omega}, P_{\omega+1}, \ldots, P_{\xi}, \ldots,
$$

formée de tous les ensembles linéaires parfaits.

Soit (pour tout $\alpha<\Omega$ ) $\Gamma_{\alpha}$ un ensemble $G_{\delta}$ de mesure nulle dense dans $P_{\alpha}$. Nous définirons maintenant par l'induction transfinie une suite transfinie $\left\{E_{\alpha}\right\}_{\alpha<\Omega}$ d'ensembles linéaires parfaits de mesure nulle comme il suit.

Soit $E_{1}$ un ensemble parfait de mesure nulle donné quelconque.

1) Pour qu'un ensemble $E$ jouisse de la propriété de Baire (au sens restreint) il faut et il suffit qa'on ait pour tout ensemble parfait $P$ la formule $E P=Q+K$, oú $Q$ est un ensemble mesarable $B$ et $K$ est un ensemble de $1^{\text {ro }}$ catégorio relativemont a $P$.

$\left.{ }^{2}\right)$ c. à. d. do $1^{\text {re }}$ catégorie sur tout ensemble parfait ( $\mathrm{L} n$ sin) 